\title{
Optogenetic Activation of Septal Glutamatergic Neurons Drive Hippocampal Theta Rhythms
}

\author{
ㄱennifer Robinson, Frédéric Manseau, Guillaume Ducharme, Bénédicte Amilhon, Erika Vigneault, \\ Salah El Mestikawy, and @Sylvain Williams \\ Douglas Mental Health University Institute, McGill University, Department of Psychiatry, Montréal, Québec H4H 1R3, Canada
}

The medial septum and diagonal band of Broca (MS-DBB) has an essential role for theta rhythm generation in the hippocampus and is critical for learning and memory. The MS-DBB contains cholinergic, GABAergic, and recently described glutamatergic neurons, but their specific contribution to theta generation is poorly understood. Here, we examined the role of MS-DBB glutamatergic neurons in theta rhythm using optogenetic activation and electrophysiological recordings performed in in vitro preparations and in freely behaving mice. The experiments in slices suggest that MS-DBB glutamatergic neurons provide prominent excitatory inputs to a majority of local GABAergic and a minority of septal cholinergic neurons. In contrast, activation of MS-DBB glutamatergic fiber terminals in hippocampal slices elicited weak postsynaptic responses in hippocampal neurons. In the in vitro septo-hippocampal preparation, activation of MSDBB glutamatergic neurons did increase the rhythmicity of hippocampal theta oscillations, whereas stimulation of septo-hippocampal glutamatergic fibers in the fornix did not have an effect. In freely behaving mice, activation of these neurons in the MS-DBB strongly synchronized hippocampal theta rhythms over a wide range of frequencies, whereas activation of their projections to the hippocampus through fornix stimulations had no effect on theta rhythms, suggesting that MS-DBB glutamatergic neurons played a role in theta generation through local modulation of septal neurons. Together, these results provide the first evidence that MS-DBB glutamatergic neurons modulate local septal circuits, which in turn contribute to theta rhythms in the hippocampus.

Key words: glutamatergic; hippocampus; septum; theta

\section{Significance Statement}

The current paper explores the functional role of septal glutamatergic neurons, a recently identified population, in theta rhythm generation in the hippocampus. Using optogenetics and electrophysiology, we have explored the functional connectivity of these neurons in vitro, as well as their influence on theta rhythms both in vitro and in vivo, and show that this neuronal population can powerfully drive theta rhythms through intraseptal connections. These results strongly suggest a role of glutamatergic neurons in theta rhythm generation and may therefore be important for learning and memory.

\section{Introduction}

The medial septum and diagonal band of Broca (MS-DBB) is well known to be a major contributor to theta generation in the hippocampus and has an essential role in learning and memory (Winson, 1978). Recent evidence suggests that the MS-DBB provides an important input for cell assembly formation in the hip-

\footnotetext{
Received June 3, 2015; revised Jan. 22, 2016; accepted Feb. 1, 2016.

Author contributions: S.W. designed research; J.R., F.M., and E.V. performed research; B.A. and S.E.M. contributed unpublished reagents/analytic tools; J.R. and G.D. analyzed data; J.R., B.A., and S.W. wrote the paper.

This work was supported by the Canadian Institutes of Health Research and Natural Sciences and Engineering Research Council of Canada. B.A. was supported by postdoctoral fellowships from Fondation Fyssen and from Fonds de la Recherche en Santé du Québec.

The authors declare no competing financial interests.

Correspondence should be addressed to Dr. Sylvain Williams, Douglas Mental Health University Institute, McGill University, Department of Psychiatry, 6875 Lasalle Blvd, Montréal, Québec H4H 1R3, Canada. E-mail: sylvain.williams@gmail.com.

DOI:10.1523/JNEUROSCI.2141-15.2016

Copyright $\odot 2016$ the authors $\quad 0270-6474 / 16 / 363016-08 \$ 15.00 / 0$
}

pocampus and grid cell organization in the entorhinal cortex (Brandon et al., 2011; Wang et al., 2015). Whereas the traditional model of the septum consists of GABAergic and cholinergic neurons, a third population of glutamatergic neurons expressing Type 2 vesicular glutamate transporters (VGLUT2) was described more recently (Sotty et al., 2003; Manseau et al., 2005). MS-DBB VGLUT2 neurons make up 25\% of the neural population of this area (Colom et al., 2005) and provide both local and septo-hippocampal projections (Colom et al., 2005; Manseau et al., 2005; Henderson et al., 2010; Huh et al., 2010). Glutamatergic neurons display a heterogeneous firing pattern, including fast, slow, burst, and cluster-firing properties in slice (Sotty et al., 2003; Huh et al., 2010), providing functional excitatory input to both cholinergic and GABAergic neurons within the septum (Manseau et al., 2005), and sending direct projections to a restricted number of pyramidal cells and interneurons in the hippocampus (Huh et al., 2010; Sun et al., 2014). 
In this study, we explored the functional connections of MSDBB glutamatergic neurons with neurons of the septum and hippocampus, and studied their role in theta generation. To determine this, we used optogenetics to control MS-DBB glutamatergic neuron activity in vitro and in vivo. We demonstrate that these neurons provide dense connections within the septum and comparatively sparse projections across the hippocampus. Using the in vitro intact septo-hippocampal preparation (Manseau et al., 2008), we show that VGLUT2 neurons modulate the rhythmicity of spontaneously generated theta rhythm through a mechanism dependent on local septal connections. Finally, we show that rhythmic activation of septal glutamatergic neurons can powerfully drive hippocampal rhythms through local septal interactions rather than through direct projections to the hippocampus.

\section{Materials and Methods}

Virus injections. All procedures were performed according to protocols and guidelines approved by the McGill University Animal Care Committee and the Canadian Council on Animal Care. VGLUT2-Cre knock-in homozygote mice (The Jackson Laboratory, stock \#016963) were housed in a 12:12 h light/dark cycle with food and water ad libitum.

To trigger the expression of excitatory opsin ChETA $_{\text {TC }}$, VGLUT2Cre mice of either sex were stereotaxically injected in the septum with a Cre-dependent adeno-associated viral vector (AAVdj-Ef1 $\alpha$-DIOhChR2(E123T/T159C)-eYFP from Molecular Virology Support Core, Oregon Health and Science University or Stanford Neuroscience Gene Vector and Virus Core, Stanford University). Control experiments were performed with either Cre-dependent eYFP control virus (AAVdj-Ef1 $\alpha$ Flex-eYFP from Vollum Vector Core, Oregon Health and Science University) or the nonspecific synapsin promoter-ChR2 virus (AAV2hSyn-ChR2(H134R)-eYFP from Canadian Neurophotonic Platform, Laval University). In addition, a subset of experiments used Credependent retrograde virus AAV2/9-hChR2 injected in the hippocampus (AAV2/9.EF1a.DIO.hChR2(H134R)-eYFP.WPRE.hGH from Penn Vector Core, University of Pennsylvania).

For all in vitro experiments, VGLUT2-Cre mouse pups were injected on postnatal day 15 (P15), whereas for in vivo experiments, adult mice were injected at $\sim 10$ weeks of age. Mice were anesthetized using isoflurane and positioned in a stereotaxic frame (Stoelting), and viruses were delivered into the septum $(0.6 \mu \mathrm{l}$ at $0.06 \mu \mathrm{l} / \mathrm{min})$. Pups were injected as follows: anteroposterior (AP) $0.86 \mathrm{~mm}$ from bregma, mediolateral (ML) $0.5 \mathrm{~mm}$, dorsoventral (DV) $-4.53 \mathrm{~mm}$ at a $4.5^{\circ}$ angle. Adults were injected as follows: AP $0.8 \mathrm{~mm}$ from bregma, ML $0.5 \mathrm{~mm}$, DV $-4.5 \mathrm{~mm}$ at a $5^{\circ}$ angle. For tracer experiments, the retrograde virus AAV2/9-hChR2 was injected at four injection sites bilaterally across the hippocampus: (1) $0.3 \mu \mathrm{l}$ at $\mathrm{AP}-1.82 \mathrm{~mm}$ from bregma, $\mathrm{ML} \pm 1.5 \mathrm{~mm}, \mathrm{DV}-1.5 \mathrm{~mm}$; (2) $0.3 \mu \mathrm{l}$ at $\mathrm{AP}-2.45 \mathrm{~mm}, \mathrm{ML} \pm 2.5 \mathrm{~mm}, \mathrm{DV}-1.5 \mathrm{~mm}$; (3) $0.2 \mu \mathrm{l}$ at AP $-3.52 \mathrm{~mm}, \mathrm{ML} \pm 3.25 \mathrm{~mm}, \mathrm{DV}-3.42 \mathrm{~mm}$; and (4) $0.2 \mu \mathrm{l}$ at AP -3.52 $\mathrm{mm}, \mathrm{ML} \pm 3.25 \mathrm{~mm}, \mathrm{DV}-4.17 \mathrm{~mm}$.

Anatomical characterization. To quantify the specificity of virus expression in the septum, VGLUT2 mRNA was compared with eYFP expression through fluorescent in situ hybridization (FISH) and immunocytochemistry staining for eYFP. For these experiments, animals were injected with AAVdj-ChETA-eYFP virus and euthanized at 3-5 weeks following injections; two animals at P15 and three adult animals were used. Mice were deeply anesthetized; fresh brains were extracted and frozen in isopentane at $-30^{\circ} \mathrm{C}$. Coronal sections $(14 \mu \mathrm{m})$ of the septum were cut using a cryostat at $-20^{\circ} \mathrm{C}$ and mounted directly on glass slides. Antisense cRNA riboprobes were obtained from transcription of a PCR template, amplified with primers containing the T7 promoter followed by the VGLUT2 sequence (forward primer $5^{\prime}$-AATAACCCTCACTAAA GGGAGAAGACTATGCGCAGAACTCGCCT3' and reverse primer 5' TAATACGACTCACTATAGGGAGACCCGGTTAGCAGCCAGTC-3'). VGLUT2 riboprobes were labeled with UTPs coupled to digoxigenin (Roche Applied Science). Sections were fixed with $4 \%$ formaldehyde and hybridized as previously described (Gras et al., 2002). The slices were then incubated with anti-DIG coupled with HRP (1:2500, Roche Applied Science) for $1 \mathrm{~h}$ at room temperature. The TSA-plus-Cyanine 3 kit (PerkinElmer) was used to detect VGLUT2 transcript under $555 \mathrm{~nm}$ excitation fluorescence light. After mRNA detection, virus expression was identified by immunocytochemistry with GFP rabbit antiserum (36 $\mathrm{h}$ at room temperature, 1:1000, Invitrogen), followed by detection with anti-rabbit IgG coupled to AlexaFluor 488 for $1 \mathrm{~h}$ at room temperature (1:1000, Invitrogen). The slices were mounted with Fluoromount-G (Southern Biotechnology) and analyzed with an AxioObserver.Z1 microscope (Carl Zeiss).

Immunocytochemistry was used to assess virus distribution across the septum and to characterize projections to the hippocampus. Mice were perfused and sectioned as described previously (Amilhon et al., 2015). Free-floating sections across the septum and hippocampus incubated in GFP rabbit antiserum (1:1000, Invitrogen) and either ChAT goat antiserum (1:250, Millipore), parvalbumin (PV) mouse monoclonal antiserum (1:1000, Sigma-Aldrich), VGLUT2 guinea pig antiserum (1: 1000, Millipore), or Neuronal Nuclear antibody NeuN mouse monoclonal antiserum (1:1000 Millipore). Primary antibodies were detected with anti-mouse IgG1, anti-rabbit, anti-guinea pig, or anti-goat coupled to AlexaFluor-488, -555 , or -633 (1:1000, Invitrogen). Sections were mounted and analyzed across either the septum or hippocampus. Images were counted using ImageJ software (ImageJ, http://imagej.nih. gov/ij/).

In vitro experiments. Patch-clamp recordings: VGLUT2-Cre mice injected with AAVdj-ChETA were euthanized at P33 to P40 (18-25 d after viral injection). Animals were decapitated and brains extracted in cold sucrose solution containing the following (in $\mathrm{mm}$ ): 252 sucrose, 24 $\mathrm{NaHCO}_{3}, 10$ glucose, $3 \mathrm{KCl}, 2 \mathrm{MgSO}_{4} 1.25 \mathrm{NaH}_{2} \mathrm{PO}_{4}, 1.2 \mathrm{CaCl}_{2}, \mathrm{pH}$ 7.4, continuously oxygenated with $95 \% \mathrm{O}_{2} / 5 \% \mathrm{CO}_{2}$. Coronal sections of the septum (350 $\mu \mathrm{m}$ thick) or hippocampus ( $400 \mu \mathrm{m}$ thick) were sliced and recorded using the same protocol as previously published (Huh et al., 2010). Patch glass micropipettes (Warner Instruments) with a resistance 2.2-5 $\mathrm{M} \Omega$ were used, and intrapipette solution contained the following (in mM): $144 \mathrm{~K}$-gluconate, $10 \mathrm{HEPES}, 3 \mathrm{MgCl}_{2}, 2 \mathrm{Na}_{2} \mathrm{ATP}, 0.3 \mathrm{GTP}, 0.2$ EGTA, adjusted to $\mathrm{pH} 7.2$ with $\mathrm{KOH}$.

In the recording bath, sections were perfused with aCSF containing the following (in mM): $125 \mathrm{NaCl}, 26 \mathrm{NaHCO}_{3}, 25$ glucose, $2.5 \mathrm{KCl}, 2 \mathrm{MgSO}_{4}$ $1.25 \mathrm{NaH}_{2} \mathrm{PO}_{4}, 2 \mathrm{CaCl}_{2}$, pH 7.3, continuously oxygenated with $95 \%$ $\mathrm{O}_{2} / 5 \% \mathrm{CO}_{2}$, at $\sim 5 \mathrm{ml} / \mathrm{min}$ at $25^{\circ} \mathrm{C}$. For postsynaptic responses in septal and hippocampal sections, eYFP-negative neurons were targeted within the fluorescent zone. Recordings were done in current clamp with cells held at $-70 \mathrm{mV}$ and just below action potential threshold at $\sim-55 \mathrm{mV}$. EPSP amplitudes were measured with stimulations of 5 and $10 \mathrm{~ms}$ square light pulses.

Light activation was achieved using blue light ( $473 \mathrm{~nm}$ ) with a custommade LED system, consisting of LEDs (Luxeon) coupled with 1- or 3-mm-diameter light guide for light delivery (Edmund Optics).

Septo-hippocampal preparation. In these experiments, the virus and experimental timeline were the same as detailed above. The intact septohippocampal preparation was dissected as described previously (Manseau et al., 2008) and recordings done as described by Amilhon et al. (2015). Glutamatergic septal neurons were stimulated with a light guide placed above the septum; to target septal glutamatergic fibers projecting to the hippocampus, the light guide was placed above the fornix and the septum was removed. Recording electrodes were positioned in the hippocampus across the CA1/SUB temporal region. Recording protocols consisted of $60 \mathrm{~s}$ baseline, $30 \mathrm{~s}$ light stimulation, and $60 \mathrm{~s}$ postmonitoring periods. Light stimulations consisted of light pulses of $50 \%$ duty cycle duration for theta frequencies ranging from 2 to $10 \mathrm{~Hz}$. Local field potentials were recorded with a differential AC amplifier (A-M Systems), filtered online $(0.5-600 \mathrm{~Hz})$, and sampled at $5 \mathrm{kHz}$.

In vivo experiments. Mice were injected at $\sim 10$ weeks of age and, following a 3 to 4 week incubation period, were implanted with an optic fiber (Thorlabs) connected to a ferrule (Precision Fiber Products), which was implanted at a $5^{\circ}$ angle to target slightly above the medial septum (AP $0.86, \mathrm{ML}-0.2, \mathrm{DV}-3.83 \mathrm{~mm}$ ) or above the fornix at an $11^{\circ}$ angle (AP $-0.34, \mathrm{ML} 0.1, \mathrm{DV}-1.9 \mathrm{~mm})$. Animals were implanted with a headstage (EIB-18, Neuralynx), which included an electrode array constructed 
A

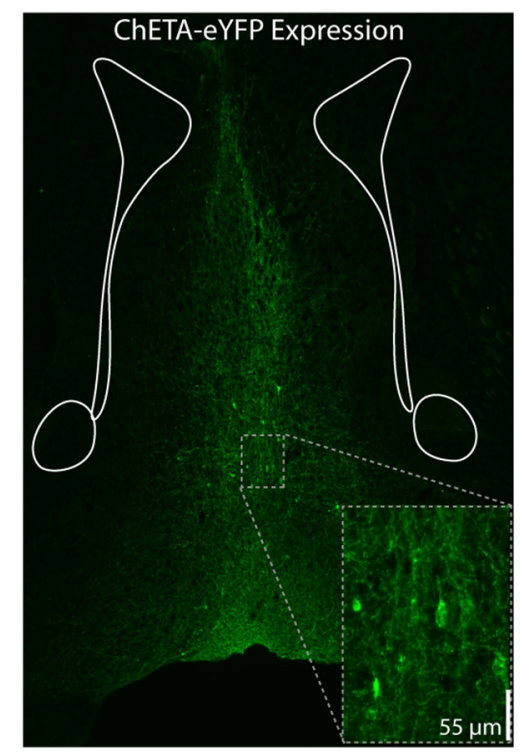

B

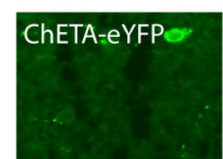

VGLUT2-mRNA
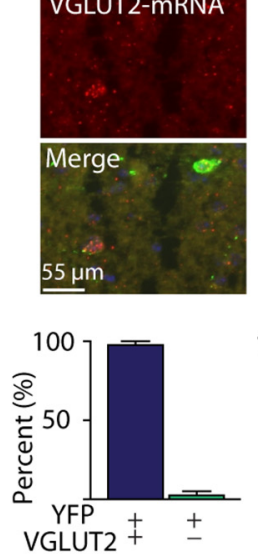

2
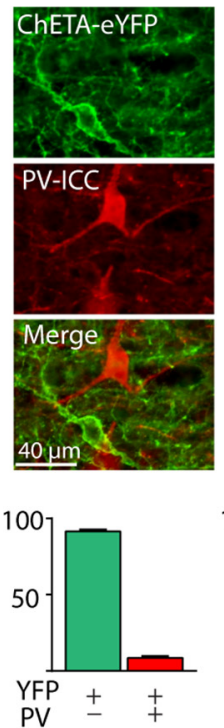

3
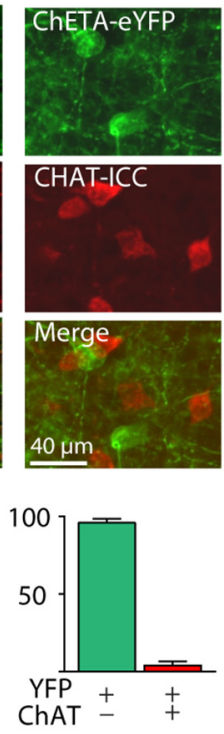

C

D Putative GABAergic

Septum Slice
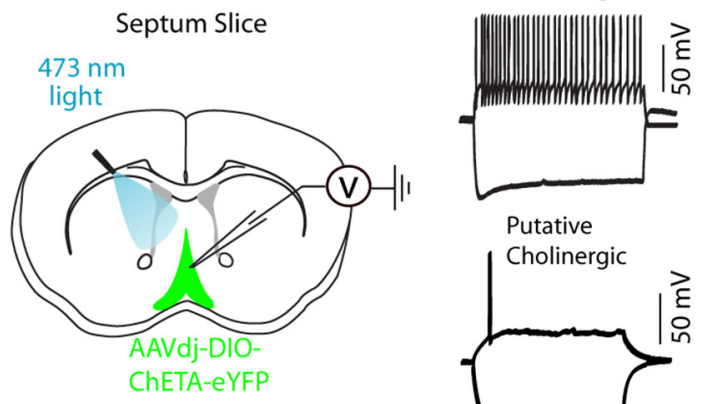

Putative

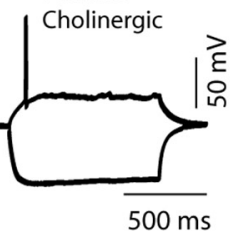

E
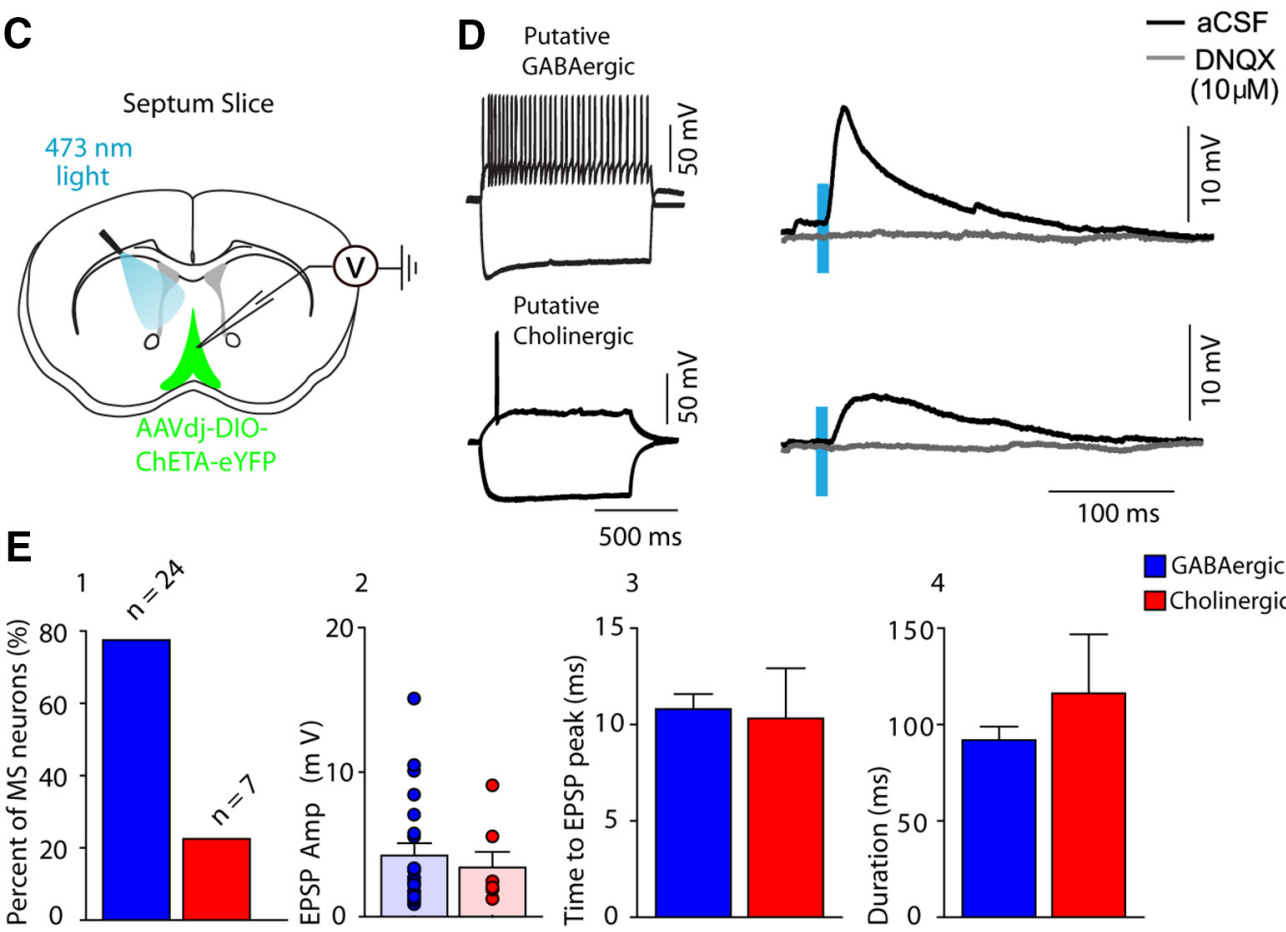

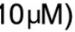
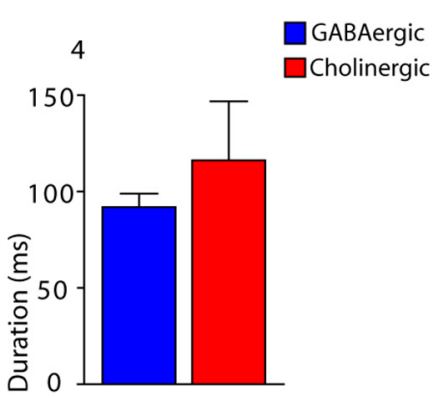

Figure 1. Optogenetics and MS-DBB glutamatergic neurons. $A$, Coronal section showing ChETA-eYFP expression from MD-DBB VGLUT2 neurons and fibers. B, ChETA-eYFP expression compared with the following: $\boldsymbol{B} 1$, VGLUT2 mRNA (of 335 neurons, eYFP/VGLUT2 mRNA: $96.8 \pm 1.3 \%$, eYFP only: $3.3 \pm 1.3 \%$ ); $\boldsymbol{B 2}$, PV (751 neurons, eYFP-positive: $91.5 \pm 1.3 \%$, positive for both markers: $8.6 \pm 1.6 \%) ; B 3$, ChAT, (576 neurons counted, eYFP-positive: $95.9 \pm 2.6 \%$, positive for both markers: $4.1 \pm 2.6 \%)$. C, Diagram representation of experimental setup for patch-clamp recordings in septum slice. $D$, Cell characterization of ChETA-negative neuron (left) and EPSP in response to $10 \mathrm{~ms}$ duration light pulses in either regular aCSF or DNQX (10 $\mu \mathrm{M})$. E1, Percentage of MS-DBB neurons patched and sorted by firing properties (either putative GABAergic or cholinergic). E2, EPSP amplitude for each cell type. E3, Time to peak. E4, EPSP duration.

from tungsten wire (A-M Systems), across two locations in CA1 (AP $-2.18, \mathrm{ML}+2.05, \mathrm{DV}-1.5 \mathrm{~mm})$ and $(\mathrm{AP}-2.59, \mathrm{ML}+1.78, \mathrm{DV}-1.5$ $\mathrm{mm}$ ). In addition, animals were implanted with two EMGs that were placed in the neck muscles. Screws placed in the skull above the frontal cortex and cerebellum served as ground and reference, respectively. Headstage and optic implants were secured to the skull with metabond and dental cement (Patterson Dental). Following surgery, animals had 1 week of recovery followed by a 1 week habituation period. Once habituated, animals were connected to custom-built headstage preamplifier tethers (Neuralynx) and an optic fiber patch cord (Thorlabs). Light delivery was achieved through an optic fiber patch cord and sleeve coupled to a $473 \mathrm{~nm}$ laser (LaserGlow), and light intensity was set for $\sim 20 \mathrm{~mW}$.
Neural recordings were amplified using a headstage preamplifier (HS-18, Neuralynx) before being digitized at $16000 \mathrm{~Hz}$ using a Digital Lynx SX recording system (Neuralynx) and stored with Cheetah Software (Neuralynx). Animals were recorded both in home cage and in an open field ( $44 \times 44 \mathrm{~cm}$ square box). Recording protocols took place during walking/running with the stimulation protocol consisting of a $20 \mathrm{~s}$ baseline, $20 \mathrm{~s}$ periodic stimulations within the theta range and $20 \mathrm{~s}$ after recording. Following the experiments, animals were perfused and brains were processed as described previously (Amilhon et al., 2015). Brains were sectioned on the cryostat $(35 \mu \mathrm{m})$ with every second section mounted on superfrost plus slides, which were stained for cresyl violet to identify electrode positioning within CA1 and optic fiber tracts. 

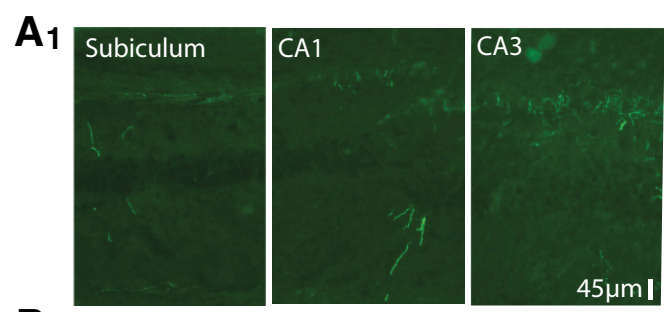

B1
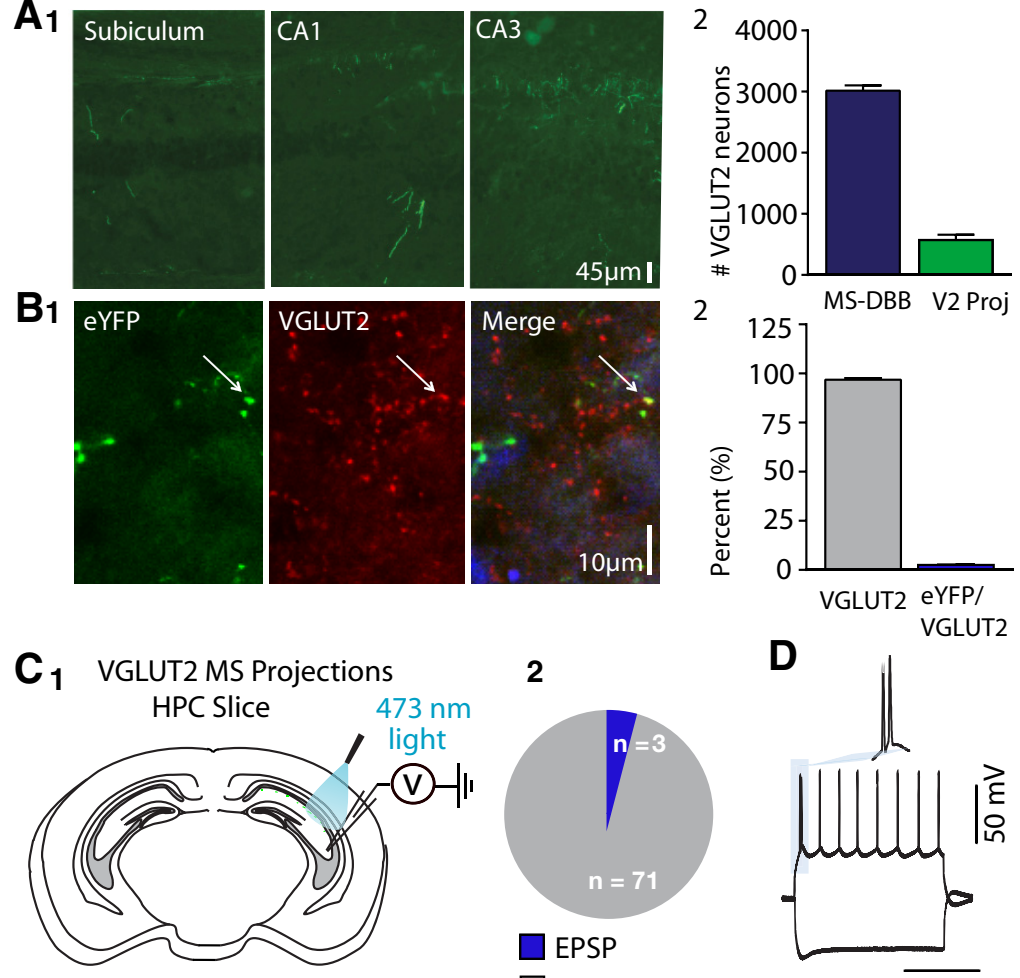

2
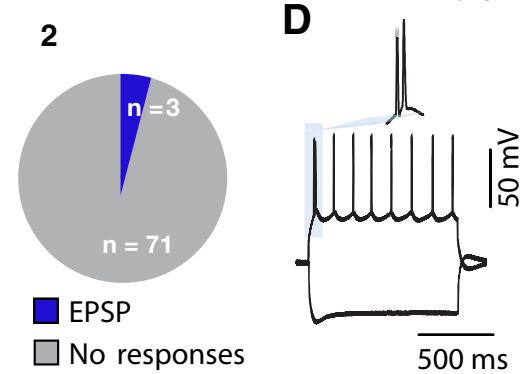

E CA3 Strat. Oriens Interneuron
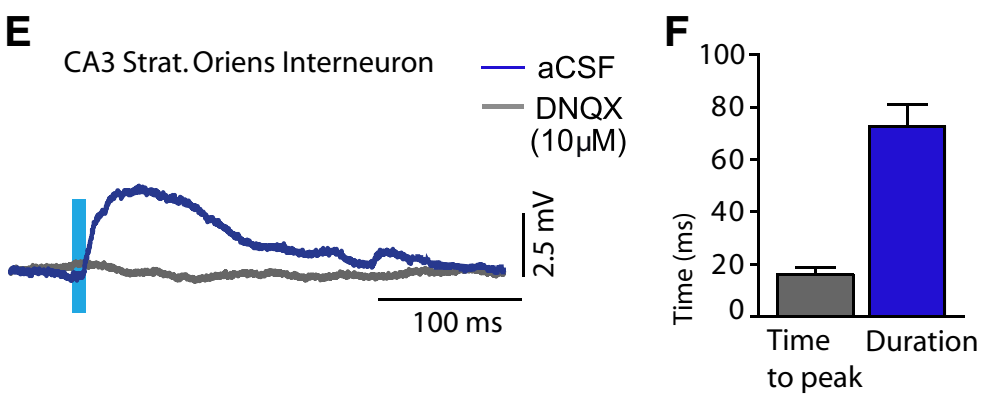

Figure 2. Characterization of MS-DBB glutamatergic hippocampal projections. A1, Coronal section showing eYFP expression of fiber projections from MS-DBB VGLUT2 neurons across the hippocampus. A2, Proportion of MS-DBB neurons projecting to the hippocampus. B1, Coronal section showing ChETA-eYFP fiber expression and VGLUT2. B2, ChETA-eYFP fiber expression compared with total VGLUT2 terminals in the stratum oriens of CA1 and CA3 of the hippocampus. C1, Diagram representation of experimental setup for patch-clamp recordings and optogenetics in hippocampus slices. C2, Proportion of neurons recorded in hippocampus displaying light-activated glutamatergic EPSPS. D, Cell characterization of hippocampal neuron. $\boldsymbol{E}$, EPSP in response to $10 \mathrm{~ms}$ light pulses in either regular aCSF or DNQX (10 $\mu \mathrm{m}) . \boldsymbol{F}$, Light-induced EPSP time to peak (ms) and EPSP duration for hippocampal neurons.

Data analysis. Patch-clamp recordings were analyzed using Clampfit 9 (Molecular Devices). In vitro septo-hippocampal preparation and in vivo field recordings were analyzed using custom MATLAB scripts (The MathWorks). For in vitro septo-hippocampal preparation field recordings, $30 \mathrm{~s}$ episodes were analyzed during baseline, stimulation, and poststimulation segments. Spectral analysis was performed using a multitaper fast Fourier transform (Chronux package, window $=5 \mathrm{~s}$, step $=0.5 \mathrm{~s}$, tapers $[1,1])$ (Bokil et al., 2010). Theta peak frequency and peak power (power at the peak frequency) were extracted from the power spectrum. Oscillation strength was used as an index of rhythmicity and was derived from the autocorrelogram (peak value of the power spectrum of the autocorrelation). Quantifications of the change in frequency, power, and oscillation strength were expressed as the percentage of change from mean of the baseline and poststimulation values. All representative traces shown in figures were filtered at $0.5-55 \mathrm{~Hz}$.

For in vivo recordings, 2-5 repetitions of 10-20 s segments were extracted for analysis. Spectral analysis was performed as described for in vitro field recordings. In vivo data was filtered off-line at $3-55 \mathrm{~Hz}$, and spectral analysis was performed using window size $=2 \mathrm{~s}$, step size $=0.5 \mathrm{~s}$. Changes in power and rhythmicity were expressed as a ratio compared with baseline values.

Statistical analysis was performed using Prism 6 (Graph Pad). Statistical analysis was performed using $t$ test, unless otherwise described. In all figures, data are mean \pm SEM.

\section{Results}

To characterize glutamatergic neurons of the MS-DBB in VGLUT2-Cre mice transfected with ChETA-eYFP, we first examined virus distribution, specificity, and coexpression with other cell type markers within the septum. Virus expression, as indicated by eYFP labeling of cell bodies, was distributed extensively across the MS-DBB (Fig. 1A). We found that the eYFP reporter was strongly expressed in VGLUT2 neurons as $96.8 \pm 1.3 \%$ of cells positive for VGLUT2 mRNA also coexpressed eYFP (total number of cells counted $=335, n=5$ mice). Nonspecific expression was very limited because only $3.3 \pm 1.3 \%$ of eYFP-positive neurons did not express VGLUT2 mRNA (Fig. 1B1; $n=5$ ). It has previously been shown that some septal neurons coexpress mRNAs for cholinergic and glutamatergic markers (Sotty et al., 2003). We therefore quantified the extent of ChETA expression in cholinergic and GABAergic cell populations in the MS-DBB using ChAT and PV immunocytochemistry. Coexpression was low because only $8.6 \pm 2.2 \%$ of PV neurons and $4.1 \pm 2.7 \%$ of ChAT neurons were also positive for eYFP, respectively (Fig. $1 B 2, B 3 ; n=3$ ).

We next determined whether optogenetic activation of VGLUT2 neurons elicitedEPSPsinnonglutamatergic(ChETAYFP negative) MS-DBB neurons recorded in whole-cell patch configuration (Sotty et al., 2003; Manseau et al., 2005; Fig. $1 C, D)$. The majority of recorded cells showing postsynaptic responses were fastfiring putative GABAergic neurons (24 of 31 neurons), whereas fewer slow-firing putative cholinergic neuron responses were recorded (7 of 31 neurons) (Fig. 1E1). The amplitude of EPSPs for GABAergic and cholinergic neurons was similar ( $4.5 \pm 0.9$ and $3.6 \pm 1.2 \mathrm{mV}$, respectively) (Fig. 1E2), and no significant differences were found in EPSP characteristics between GABAergic and cholinergic cells in terms of either time to peak (Fig. 1E3) or duration (Fig. 1E4). Optogenetic activation of VGLUT2 neurons did, however, drive spiking in 5 of 24 GABAergic neurons, whereas cholinergic neurons never displayed firing. In all cases, bath application of the AMPA receptor antagonist DNQX $(10 \mu \mathrm{M})$ completely blocked light-evoked EPSPs $(n=6)$ (Fig. 1D, right).

Next, we sought to characterize septal glutamatergic projections to the hippocampus. Overall, we found that glutamatergic 
A
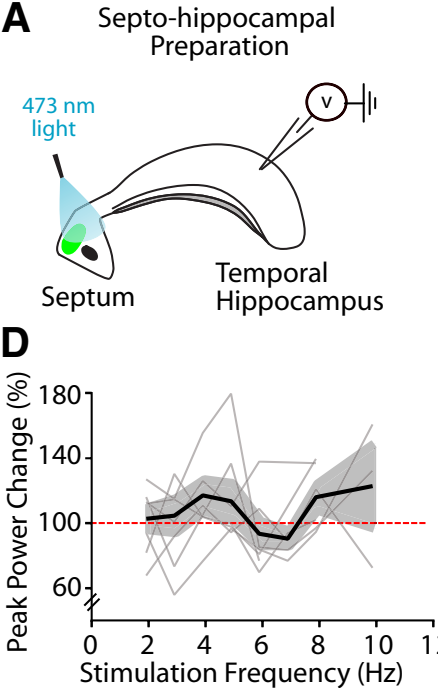

G

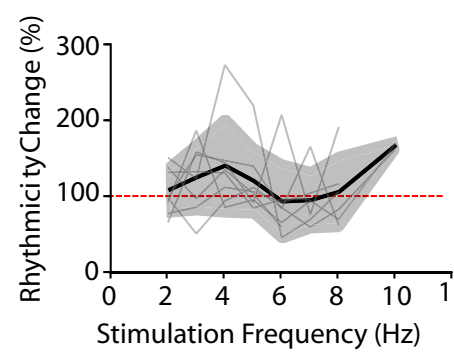

B

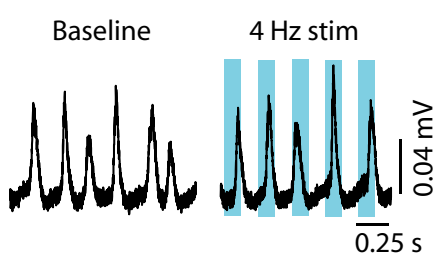

C
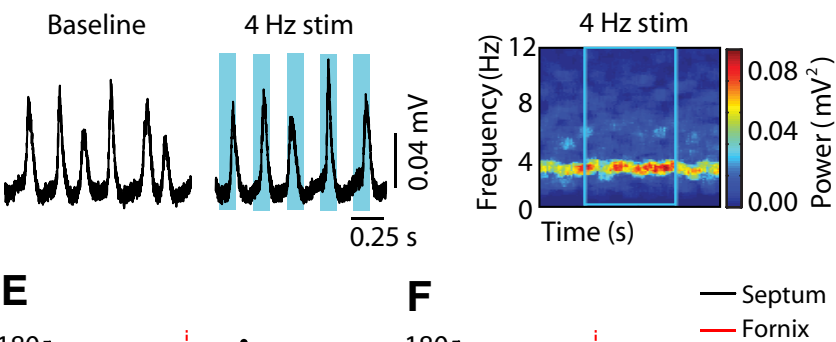

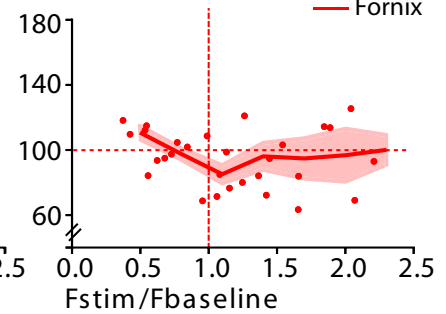

H

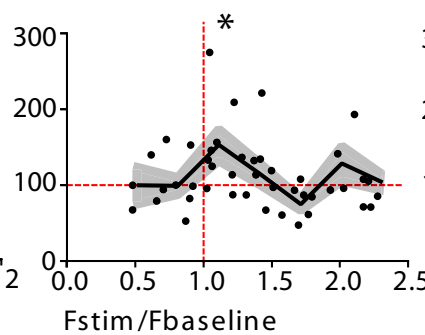

I

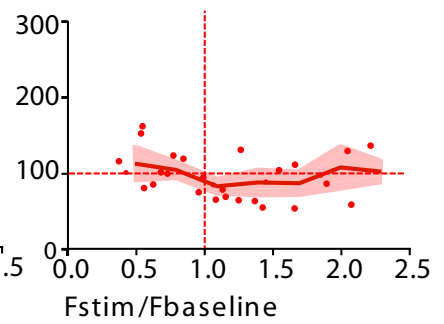

Figure 3. MS-DBB glutamatergic neurons drive hippocampal theta oscillations in vitro. $\boldsymbol{A}$, Diagram representation of experimental setup with septum light stimulations. $\boldsymbol{B}$, Example trace before and during $4 \mathrm{~Hz}$ stimulation. C, Corresponding spectrogram. D, Change in peak power (stimulation/mean of pre-post) for septal stimulations from 2 and $10 \mathrm{~Hz}$. Thin lines indicate individual preparations. Thick line indicates average. $\boldsymbol{E}$, Change in peak power (stimulation/mean pre-post) for septal stimulations expressed as a function of frequency of stimulation/frequency of baseline. Thick line indicates mean of binned data (bin number $=7$, bin size $=0.3$ ). $\boldsymbol{F}$, Change in peak power for fornix stimulations expressed as a function of frequency of stimulation/frequency of baseline (same bin size used in $\boldsymbol{E}$ ). $\boldsymbol{G}$, Change in rhythmicity (stimulation/mean baseline and poststimulation) for septal stimulation from 2 to $10 \mathrm{~Hz}$. Thin lines indicate individual preparations. Thick line indicates average. $\boldsymbol{H}$, Change in rhythmicity (stimulation/mean pre-post) for septal stimulations expressed as a function of frequency of stimulation/frequency of baseline (same bin size used in $\boldsymbol{E}$ ). $\boldsymbol{I}$, Change in rhythmicity (stimulation/mean pre-post) for fornix stimulations expressed as a function of frequency of stimulation/frequency of baseline (same bin size used in $\boldsymbol{E}$ ). ${ }^{*} p<0.05$.

MS-DBB projections were sparse across the hippocampus (Fig. 2A1). To quantify septal glutamatergic projections, we compared the total number of MS-DBB glutamatergic neurons with those projecting to the hippocampus. The total MS-DBB glutamatergic population was determined by quantifying ChETAeYFP-expressing VGLUT2 somas across the entire septum $(n=$ 2 ). The proportion of glutamatergic neurons projecting to the hippocampus was assessed by quantifying, across the entire septum, the number of VGLUT2 somas that were transfected with the retrograde tracer AAV2/9-hChR2 injected across the hippocampus $(n=3)$. The number of MS-DBB glutamatergic neurons projecting to the hippocampus was comparatively low, with only $573 \pm 84.0$ projecting neurons of $3015 \pm 85.5$ VGLUT2 neurons found across the entire septum (Fig. 2A2). To determine the proportion of VGLUT2 terminals in the hippocampus that originate from the medial septum, we quantified the number of immunolabeled VGLUT2 terminals that colocalized with MSDBB VGLUT2-eYFP-positive projections, and found a very low proportion of VGLUT2 terminals (total number of terminals counted $=3710, n=3$ mice, $2.6 \pm 0.2 \%$; Fig. $2 B 1, B 2$ ). Despite the low level of projections to the hippocampus, MS-DBB glutamatergic neurons did show some functional connections to hippocampal neurons. Whole-cell recordings of neurons located in or near stratum oriens displayed postsynaptic potentials elicited from ChETA-expressing MS-DBB VGLUT2 projections (Fig.
2C1). EPSPs were recorded in only 3 of 74 hippocampal neurons (Fig. 2C2), and their amplitude was small (1.4 $\pm 1.0 \mathrm{mV})$. The delay between light onset and the peak of postsynaptic response was on average $16.0 \pm 4.7 \mathrm{~ms}$, and the total duration of responses was $72.5 \pm 14.3 \mathrm{~ms}$ (Fig. $2 F$ ). These EPSPs were completely blocked by DNQX (10 $\mu \mathrm{M})$ (Fig. $2 E)$. Overall, these results suggest that $\mathrm{MS}-\mathrm{DBB}$ glutamatergic neurons have strong connections within the septum but few functional connections within the hippocampus.

To determine whether glutamatergic neurons from the MSDBB could drive hippocampal networks at theta frequencies, we activated ChETA-expressing VGLUT2 neurons of the MS-DBB while recording spontaneously generated theta oscillations in the isolated septo-hippocampal preparation (Fig. $3 A$ ). It was observed that optogenetic activation of MS-DBB VGLUT2 neurons was able to drive endogenous hippocampal rhythms (Fig. $3 B, C$ ). Activation of VGLUT2 neurons in the MS-DBB within theta range $(2-10 \mathrm{~Hz})$ did not significantly affect the power or rhythmicity (oscillation strength; see Materials and Methods) of the ongoing oscillation, although a high variability in the response was observed (Fig. 3D, G), which was likely due to variability in the endogenous baseline frequency. Changes in power and rhythmicity were thus expressed as a function of the frequency of the stimulation over the baseline frequency (Fstim/Fbase). A slight increase in power was observed when MS-DBB 
A

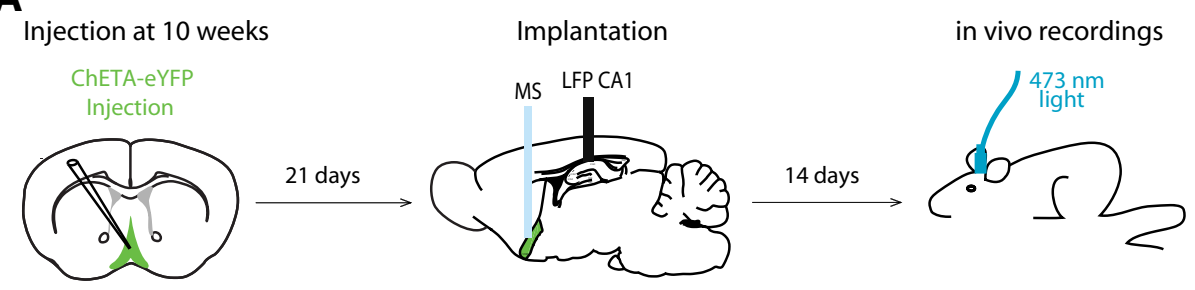

B Baseline

Septum Stimulations
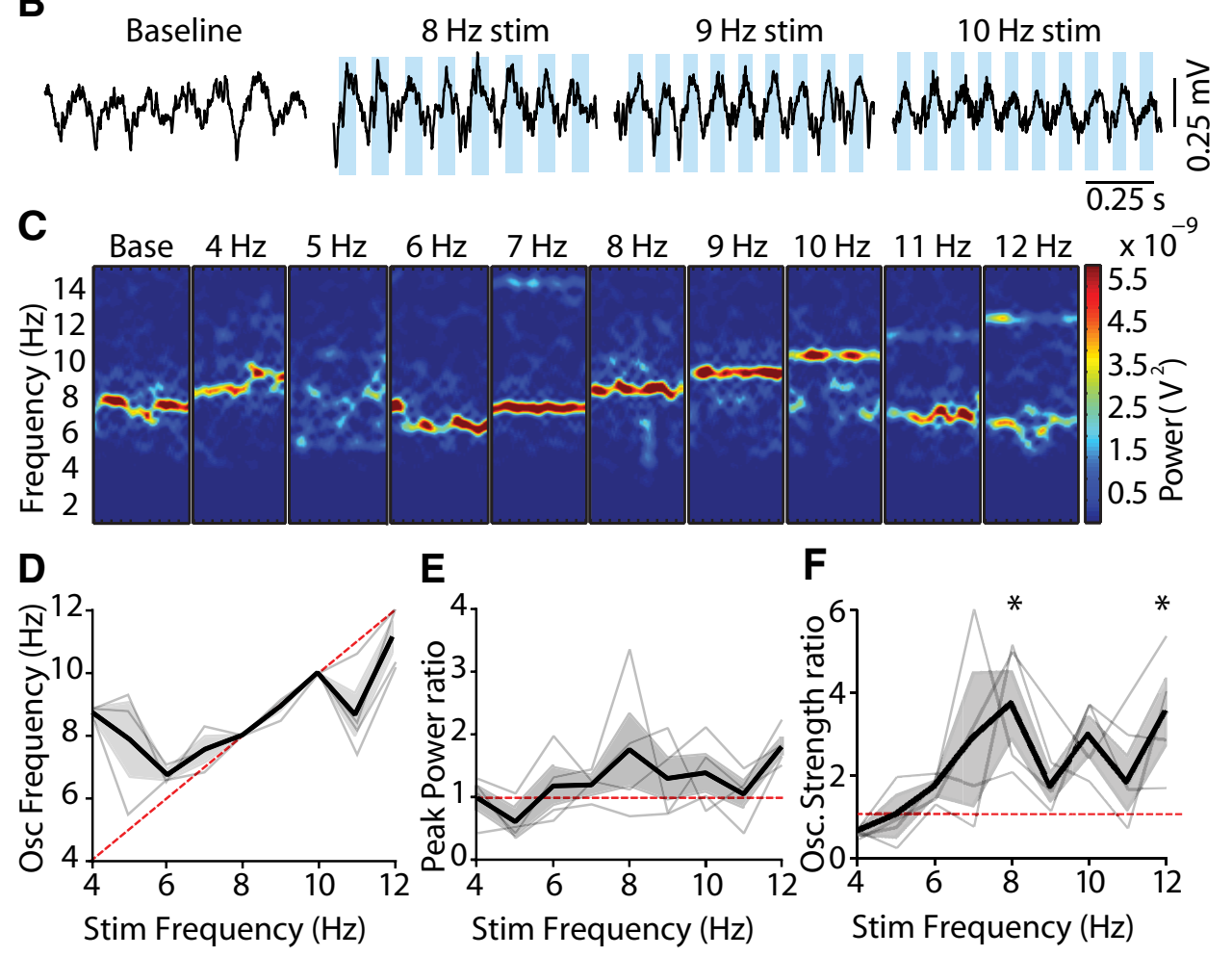

Figure 4. MS-DBB glutamatergic neurons drive hippocampal theta oscillations in vivo. $\boldsymbol{A}$, Diagram representation of experimental timeline used for in vivo experiments. $\boldsymbol{B}$, Representative traces in response to 8,9 , and $10 \mathrm{~Hz}$ stimulations. C, Example spectrograms of $10 \mathrm{~s}$ segments across stimulation frequencies for septal stimulations. $\boldsymbol{D}$, Peak frequency of oscillations expressed as a function of frequency of stimulation for septal stimulations. Thin lines indicate individual animals. Thick line indicates average. $\boldsymbol{E}$, Change in peak power ratio (stimulation/baseline) for septal stimulations from 4 to $12 \mathrm{~Hz}$. $\boldsymbol{F}$, Change in rhythmicity ratio (stimulation/baseline) for septal stimulations from 4 to $12 \mathrm{~Hz}$. ${ }^{*} p<0.05$.

VGLUT2 neurons were activated at a frequency similar or close to the baseline frequency (Fig. 3E; binned data: bin size $=0.3$, Fstim $/$ Fbase $=1.1,116.8 \pm 7.8 \%)$. A large increase was found in oscillation rhythmicity for the same range of Fstim/Fbase ratio (Fig. $3 H$; Fstim/Fbase $=1.1,153.1 \pm 22.0 \%$, $n=8, p<0.05$ ). To assess whether this effect was driven by local interactions within the MS-DBB or due to VGLUT2 projections to the hippocampus, the MS-DBB was removed from the preparation and ChETA-expressing projections were activated at the level of the fornix. Interestingly, activation of ChETA-expressing VGLUT2 fibers at the level of the fornix did not modulate theta oscillations in the hippocampus. No significant changes in power or rhythmicity were found with VGLUT2 fiber activation (Fig. $3 F, I ; n=5$ ). Therefore, specific activation of MS-DBB VGLUT2 projections to the hippocampus was not sufficient to modulate theta rhythm, whereas direct activation of MS-DBB VGLUT2 neurons and presumably entrainment of local network within the MS-DBB increased the rhythmicity of theta oscillations.

Last, we assessed whether MS-DBB glutamatergic neuron activation modulated theta rhythm in vivo. Theta oscillations were recorded during voluntary movement (walking or running) and the effect of stimulating either VGLUT2 neurons in the MS-DBB or VGLUT2 projections at the level of the fornix was quantified (Figs. 4, 5). MS-DBB stimulations robustly modulated the frequency of theta rhythm within the $6-10 \mathrm{~Hz}$ range, as can be seen in the linear relationship between the frequency of stimulation and the frequency of oscillations (Fig. $4 C, D ; n=4$ ). Although oscillation frequency was tightly modulated by optogenetic stimulations during septal activation, the peak power of theta rhythm was not significantly changed (Fig. 4E). Interestingly, theta rhythmicity showed an increase at 8 and $12 \mathrm{~Hz}$ stimulations compared with baseline (Fig. $4 F ; 2.7 \pm 0.8$-fold and $2.5 \pm 0.9$-fold increase, $p<0.05)$. Therefore, activation of VGLUT2 neurons in the MS-DBB robustly modulates frequency and rhythmicity of hippocampal theta. In contrast, when targeting the VGLUT2 direct projections to the hippocampus though fornix activation, stimulations had no effect on frequency of ongoing rhythms (Fig. $5 A 1, B ; n=3$ ). In addition, power and rhythmicity of theta oscillations were also not modulated (Fig. 5C,D). To ensure that the lack of effect was not due to inefficiency of fornix stimulation, additional 

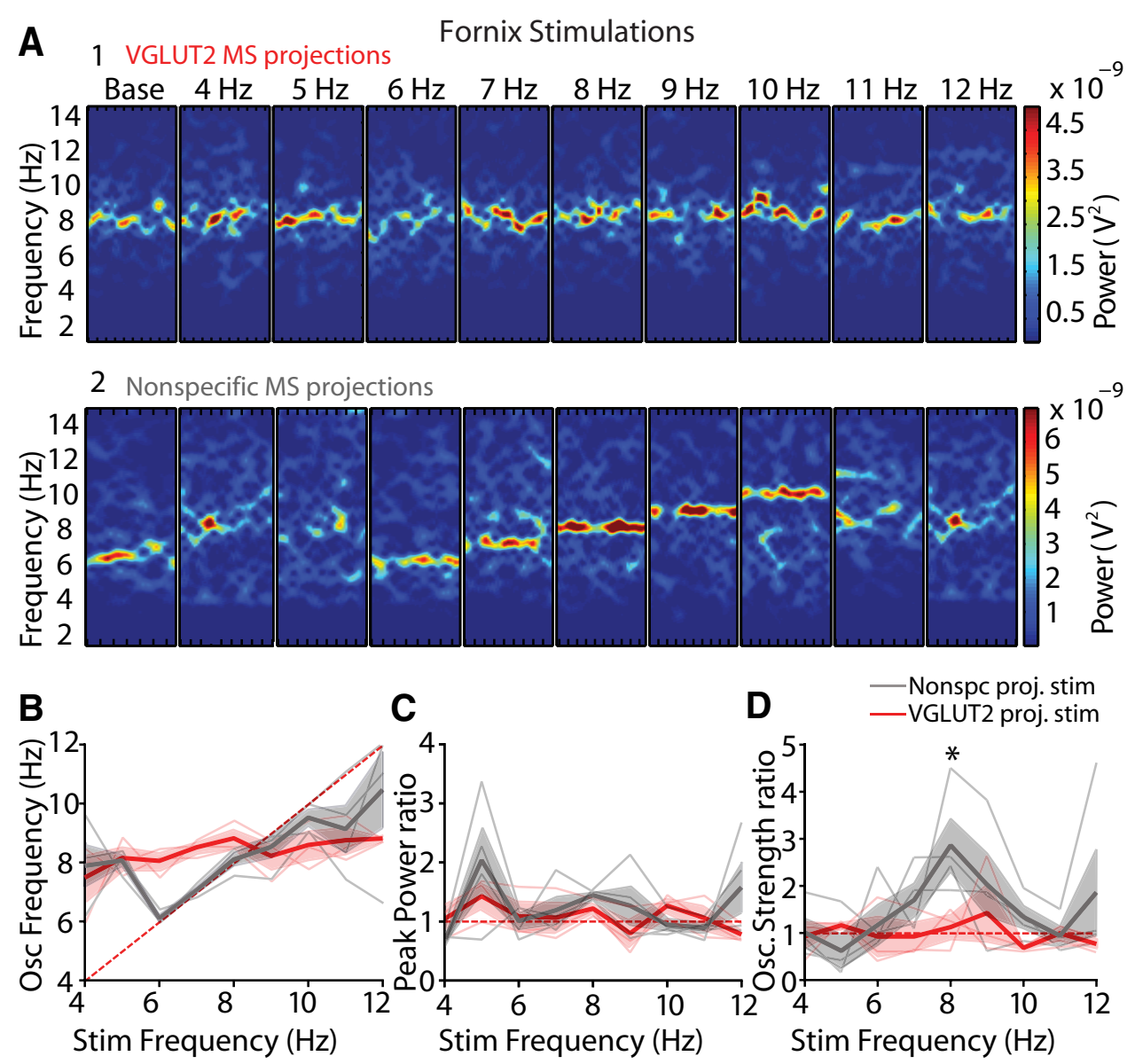

Figure 5. MS-DBB glutamatergic neuron fornix stimulation does not influence hippocampal theta oscillations in vivo. $A$, Example spectrograms of 10 s segments across stimulation frequencies for VGLUT2 fornix (A1) and nonspecific fornix (A2) stimulations. B, Peak frequency of oscillations expressed as a function of frequency of stimulation for fornix stimulations. Thin lines indicate individual animals. Thick line indicates average. C, Change in peak power ratio (stimulation/baseline) for fornix stimulations from 4 to $12 \mathrm{~Hz}$. $\boldsymbol{D}$, Change in rhythmicity ratio (stimulation/baseline) for fornix stimulations from 4 to $12 \mathrm{~Hz}$. ${ }^{*} p<0.05$.

experiments were performed on animals injected with synapsin-ChR2 virus in the MS-DBB to target all neuronal types. In these experiments, fornix stimulation strongly modulated the frequency of ongoing rhythms (Fig. $5 A 2, B ; n=4$ ). In addition, although the peak power was unaffected (Fig. $5 C$ ), there were large increases in theta rhythmicity at $8 \mathrm{~Hz}$ stimulations compared with baseline (Fig. 5D; $2.9 \pm 0.6$-fold mean increase, $p<0.05$ ). To control for the potential influence of light, additional experiments were performed on animals injected with a control virus driving the expression of eYFP alone. No changes were identified in frequency, power, or rhythmicity over the range of stimulations (data not shown, $n=2$ ).

Overall, these results show that septal glutamatergic neurons have strong excitatory connections within the MS-DBB to both GABAergic and cholinergic neurons and sparse connections to hippocampal neurons. In addition, rhythmically activating VGLUT2 neurons in the MS-DBB can drive theta oscillations, whereas stimulation of fibers projecting directly to the hippocampus through the fornix had no effect on theta rhythm. These results suggest that glutamatergic neurons can drive rhythms through intraseptal connections, activating both GABAergic and cholinergic neurons, which in turn modulate hippocampal network activity.

\section{Discussion}

The medial septum is a major input pathway to the hippocampus, contributing to hippocampal theta rhythms. Although pre- vious studies have extensively studied the role of GABAergic and cholinergic neurons in the septum, the main purpose of this study was to determine whether the third neuronal population of the septum, glutamatergic neurons, contributes to hippocampal theta rhythms. We used three approaches to address this question. We first used patch-clamp recordings in slice to examine the role of MS-DBB glutamatergic projections locally within the septum network and distally in the hippocampus. We recorded both GABAergic and cholinergic neurons within the MS-DBB and found that, although a much higher number of GABAergic neurons showed light-induced postsynaptic responses, both populations were activated reliably. Second, we used the intact septohippocampal and the isolated hippocampal preparations to examine the effect of septal and fornix stimulations on endogenous theta rhythms. This preparation provides a model to investigate septo-hippocampal networks free from external inputs (entorhinal cortex, contralateral hippocampus, and prefrontal cortex), providing evidence for the minimal network required to drive these rhythms. Our results showed that MS-DBB glutamatergic neurons can modulate hippocampal theta power and rhythmicity by their actions through the local septal network. Although MS-DBB glutamatergic neurons are known to have projections to a portion of both pyramidal neurons and interneurons in the hippocampus (Huh et al., 2010; Sun et al., 2014), these projections (isolated by fornix stimulations) were not sufficient to modulate theta oscillations. 
Last, we showed that optogenetic activation of MS-DBB glutamatergic neurons can efficiently drive theta rhythms in the behaving animal during movement. MS-DBB septal activation can tightly modulate the rhythm's frequency from 6 to $10 \mathrm{~Hz}$ and increase rhythmicity. Interestingly, activation of MS-DBB glutamatergic neurons in the isolated septo-hippocampal preparation did not show the striking frequency tuning that was observed during in vivo septum activation. This difference is likely due to additional pathways in vivo, with VGLUT2 septal neurons sending glutamatergic inputs to brain regions, which in turn influence theta rhythm in the hippocampus, but this point remains to be investigated.

Together, our results suggest that MS-DBB glutamatergic neurons take part in hippocampal rhythm generation through local connections within the septum. Recent evidence using optogenetic activation of MS-DBB cholinergic neurons suggests that ACh neurons may not significantly take part in theta generation in the hippocampus (Vandecasteele et al., 2014). As for MS-DBB GABAergic neurons, these neurons have unique connectivity to GABAergic interneurons in the hippocampus and strongly fire phase-locked to theta, suggesting that they play an important role in theta generation (Petsche et al., 1962; Freund and Antal, 1988; Stewart and Fox, 1990; Hangya et al., 2009; Bender et al., 2015). Finally, recent in vivo evidence has shown that activation of glutamatergic neurons in the MS-DBB initiates both theta rhythms and locomotor activity in the head-restrained animal (Fuhrmann et al., 2015). Results from this study suggest that direct glutamatergic MS-DBB projections drive interneurons in the stratum oriens, which in turn influence CA1 pyramidal cell firing. In comparison, the current study was performed on freely behaving animals; and although septal stimulations did appear to increase overall arousal of the animal, no velocity measurements were performed. In contrast, however, our anatomical quantification and electrophysiological data in the hippocampus suggest weak projections to the stratum oriens and do not support the paradigm that direct projections significantly drive hippocampal firing during theta rhythms. Instead, we found multiple layers of evidence supporting an intraseptal mechanism of frequency tuning of hippocampal theta rhythms. Our results strongly suggest that glutamatergic neurons primarily act to synchronize septal GABAergic and, to a minor extent, cholinergic inputs, to pace hippocampal theta rhythm. Finally, these results provide evidence that glutamatergic septal neurons play an important role in theta generation and may significantly participate in learning and memory.

\section{References}

Amilhon B, Huh CY, Manseau F, Ducharme G, Nichol H, Adamantidis A, Williams S (2015) Parvalbumin interneurons of hippocampus tune population activity at theta frequency. Neuron 86:1277-1289. CrossRef Medline

Bender F, Gorbati M, Cadavieco MC, Denisova N, Gao X, Holman C, Korotkova T, Ponomarenko A (2015) Theta oscillations regulate the speed of locomotion via a hippocampus to lateral septum pathway. Nat Commun 6:8521. CrossRef Medline

Bokil H, Andrews P, Kulkarni JE, Mehta S, Mitra PP (2010) Chronux: a platform for analyzing neural signals. J Neurosci Methods 192:146-151. CrossRef Medline

Brandon MP, Bogaard AR, Libby CP, Connerney MA, Gupta K, Hasselmo ME (2011) Reduction of theta rhythm dissociates grid cell spatial periodicity from directional tuning. Science 332:595-599. CrossRef Medline

Colom LV, Castaneda MT, Reyna T, Hernandez S, Garrido-Sanabria E (2005) Characterization of medial septal glutamatergic neurons and their projection to the hippocampus. Synapse 58:151-164. CrossRef Medline

Freund TF, Antal M (1988) GABA-containing neurons in the septum control inhibitory interneurons in the hippocampus. Nature 336:170-173. CrossRef Medline

Fuhrmann F, Justus D, Sosulina L, Kaneko H, Beutel T, Friedrichs D, Schoch S, Schwarz MK, Fuhrmann M, Remy S (2015) Locomotion, theta oscillations, and the speed correlated firing of hippocampal neurons are controlled by a medial septal glutamatergic circuit. Neuron 86:1253-1264. CrossRef Medline

Gras C, Herzog E, Bellenchi GC, Bernard V, Ravassard P, Pohl M, Gasnier B, Giros B, El Mestikawy S (2002) A third vesicular glutamate transporter expressed by cholinergic and serotoninergic neurons. J Neurosci 22: 5442-5451. Medline

Hangya B, Borhegyi Z, Szilágyi N, Freund TF, Varga V (2009) GABAergic neurons of the medial septum lead the hippocampal network during theta activity. J Neurosci 29:8094-8102. CrossRef Medline

Henderson Z, Lu CB, Janzso G, Matto N, McKinley CE, Yanagawa Y, Halasy K (2010) Distribution and role of Kv3.1b in neurons in the medial septum diagonal band complex. Neuroscience 166:952-969. CrossRef Medline

Huh CY, Goutagny R, Williams S (2010) Glutamatergic neurons of the mouse medial septum and diagonal band of Broca synaptically drive hippocampal pyramidal cells: relevance for hippocampal theta rhythm. J Neurosci 30:15951-15961. CrossRef Medline

Manseau F, Danik M, Williams S (2005) A functional glutamatergic neurone network in the medial septum and diagonal band area. J Physiol 566:865-884. CrossRef Medline

Manseau F, Goutagny R, Danik M, Williams S (2008) The hippocamposeptal pathway generates rhythmic firing of GABAergic neurons in the medial septum and diagonal bands: an investigation using a complete septo-hippocampal preparation in vitro. J Neurosci 28:4096-4107. CrossRef Medline

Petsche H, Stumpf C, Gogolak G (1962) The significance of the rabbit's septum as a relay station between the midbrain and the hippocampus: I. The control of hippocampus arousal activity by the septum cells. Electroencephalogr Clin Neurophysiol 14:202-211. CrossRef Medline

Sotty F, Danik M, Manseau F, Laplante F, Quirion R, Williams S (2003) Distinct electrophysiological properties of glutamatergic, cholinergic and GABAergic rat septo-hippocampal neurons: novel implications for hippocampal rhythmicity. J Physiol 551:927-943. CrossRef Medline

Stewart M, Fox SE (1990) Do septal neurons pace the hippocampal theta rhythm? Trends Neurosci 13:163-168. CrossRef Medline

Sun Y, Nguyen AQ, Nguyen JP, Le L, Saur D, Choi J, Callaway EM, Xu X (2014) Cell-type-specific circuit connectivity of hippocampal CA1 revealed through Cre-dependent rabies tracing. Cell Rep 7:269-280. CrossRef Medline

Vandecasteele M, Varga V, Berényi A, Papp E, Barthó P, Venance L, Freund TF, Buzsáki G (2014) Optogenetic activation of septal cholinergic neurons suppresses sharp wave ripples and enhances theta oscillations in the hippocampus. Proc Natl Acad Sci U S A 111:13535-13540. CrossRef Medline

Wang Y, Romani S, Lustig B, Leonardo A, Pastalkova E (2015) Theta sequences are essential for internally generated hippocampal firing fields. Nat Neurosci 18:282-288. CrossRef Medline

Winson J (1978) Loss of hippocampal theta rhythm results in spatial memory deficit in the rat. Science 201:160-163. CrossRef Medline 